\title{
Reflexiones fenomenológicas: más allá de la tecnificación del turismo y la mercadotecnia.
}

\author{
Phenomenological reflections: beyond the technification of \\ tourism and marketing.
}

DOI: $10.32870 /$ sincronia.axxv.n80.26b21

\section{Esteban Arias Castañeda}

Facultad de Turismo y Gastronomía. Universidad Autónoma del Estado de México. (MÉXICO).

CE: esteban.arias.castaneda@gmail.com / ORCID: 0000-0001-7068-061X

\section{Roberto Mexía Valenzuela}

Departamento de Contabilidad. Universidad de Sonora. (MÉXICO).

CE: roberto.mexia.val@gmail.com

\section{Esta obra está bajo una Licencia Creative Commons Atribución-NoComercial 4.0 Internacional}

Recibido: $11 / 02 / 2021$

Revisado: $16 / 04 / 2021$

Aprobado: 23/05/2021

\section{RESUMEN}

La esencia no es otra cosa que, además de ser un acto fenomenológico, es la interpretación y ejercicio mental. Este ejercicio responde ¿qué hace que el turismo sea turismo? Y ¿qué hace la mercadotecnia sea mercadotecnia? El objetivo es esbozar la esencia del turismo y la mercadotecnia desde reflexiones fenomenológicas. El método fenomenológico es el estudio del análisis intencional, como acto intuitivo y de reflexión. La esencia del turismo y la mercadotecnia, así como de cualquier cosa, no se limita a su mera finalidad o utilidad, es decir, supera las condiciones sine que non de la cosa. 
Palabras clave: Heidegger. Esencia. Pensar. Conocer. Proyectar.

\section{ABSTRACT}

The essence is nothing other than, in addition to being a phenomenological act, it is the interpretation and mental exercise. This exercise answers what makes tourism tourism? And what makes marketing be marketing? The aim is to try out the essence of tourism and marketing from phenomenological reflections. The phenomenological method is the study of intentional analysis, as an intuitive and reflective act. The essence of tourism and marketing, as well as of anything, is not limited to its mere purpose or utility, that is, it exceeds the sine que non conditions of the thing.

Keywords: Heidegger. Essence. Think. Know. To project.

\section{Introducción}

Hoy en día, la obtención de divisas es vital para todas las naciones, y para incrementar su número, se emplean técnicas o estrategias para conseguirlo. De este modo, los expertos en las ciencias económicas se han encargado de unir al turismo y la mercadotecnia (Altamira y Muñoz, 2007; Martos, 2015; Parra y Beltrán, 2016; Papadopoulos, 1987; Kotler, et al, 2017), donde la segunda (mercadotecnia) favorece al primero (turismo) para obtener más riquezas aprovechando los recursos del país.

Ahora bien, en lo que se refiere a campos de conocimiento, se tiene que, como ha pasado con la filosofía, que ha engendrado, a partir de lo que en su día fueran pequeños apéndices, ciencias más independientes y consolidadas, como son las matemáticas o la biología; y que disciplinas como la mercadotecnia o el turismo han sido posibilitadas hace muy poco tiempo como campos de saber de carácter multi e interdisciplinar, debido al -todavía- débil andamiaje de su propio cuerpo teórico metodológico y, por consecuencia, su dependencia de las ciencias económico-administrativas, como la comunicación o la geografía, hasta más recientemente la introducción de las neurociencias clínicas. 
En este sentido, tanto el turismo como la mercadotecnia, se han considerado campos técnicos o como medidas estratégicas de carácter rentable, minimizando el esfuerzo reflexivopensante que intente proponer argumentos ajenos al positivismo.

Con el paso del tiempo, tanto el conocimiento turístico como el de mercadotecnia se han ido alimentando de reflexiones cada vez más profundas, las cuales están intentando aportar otra visión distinta a la tradicional empírica; por lo que, el objetivo de este ensayo es esbozar la esencia del turismo y la mercadotecnia desde reflexiones fenomenológicas.

La búsqueda y propuesta de la esencia no es otra cosa que, además de ser un acto fenomenológico, es la interpretación y ejercicio mental de quien escribe este texto entorno a dos campos, de los cuales, poco a poco se ha ido ampliando su corpus teórico, dejando ver la imperiosa necesidad de recurrir a la filosofía para quitar el velo que esconden esos -todavía- aparatos monetizados, mejor conocidos como Turismo y Mercadotecnia.

Por lo que, para este ejercicio de pensar se recurre al intento de responder ¿qué hace que el turismo sea turismo? Y ¿qué hace la mercadotecnia sea mercadotecnia?, superando todo juicio superficial de condiciones sine qua non.

Es importante mencionar que hablar de esencia de estas cosas se ha convertido en un anquilamiento (en términos heideggerianos), debido a que la esencia de la cosa no llega a aparecer, es decir, según M. Heidegger (2003) no llega a lenguaje, porque para la ciencia o técnica no es relevante en tanto que no genera crecimiento económico o soluciona problemas de tipo socioeconómico.

\section{Metodología}

El método fenomenológico es el estudio del hecho llamado análisis intencional, esto quiere decir que, además de ser un acto intuitivo de esencias, se trata del ejercicio de una reflexión (San Martín, 2002). 
La premisa de este método es la ya conocida frase "vuelta a las cosas mismas", para eso, la intuición debe de tomarse como base, de modo que ésta constituya un tipo de conocimiento que pone al investigador de forma directa ante la cosa.

Cuando se habla de ir a la cosa, no se refiere a la vuelta objetiva que emplearía la ciencia, sino que se trata de la liberación de todo prejuicio para así poder llegar a la cosa, dejando que éstas se presenten o se muestren a sí mismas (San Martín, 2002).

Para cumplir con el objetivo de este estudio, se emplearán los argumentos fenomenológicos de M. Heidegger (2003) en cuanto a pensar la esencia de las cosas "únicamente llegamos a la cosa en sí cuando nuestro pensar, por lo menos, haya alcanzado primero la cosa en cuanto cosa" (Heidegger, 2003, p. 226).

El camino metodológico de la fenomenología debe ser orientado por la llamada reducción o epojé, es decir, según palabras de R. Audi (2004, p. 356), es "un cambio radical de actitud por el que el filósofo se vuelva de las cosas a sus significados", esto es, el investigador debe tomar distancia o alejarse un poco de la cosa para verlo -como a una obra de arte- sin juicios ni prejuicios o sometido a leyes inadecuadas, conduciendo así a un evento tanto destructivo como constructivo (Waldenfels, 2017).

\section{De la tecnificación del turismo y la mercadotecnia a pensar el turismo y la mercadotecnia}

En ese apartado se pretende relatar, a través de los ojos de Heidegger, el alcance de la tecnificación y la importancia de pensar. Este filósofo de origen alemán, se distingue por plantear las preguntas más relevantes para la filosofía, por ejemplo: el ser, el tiempo o la cosa.

Una de esas preguntas es por la técnica, y que él [Heidegger] resume en la manipulación, tanto de la naturaleza como de la propia humanidad. Es la técnica la que origina o crea la ciencia exacta moderna, en el entendido de que es el medio para un fin, puesto que en su estructura contiene el descanso sobre aparatos técnicos, y así mismo, al progreso de la construcción de toda clase de aparatos, los cuales se enfocan en hacer de la naturaleza la estación de servicio que sirve como fuente de energía para la técnica y la industria (Heidegger, 1955). 
Ahora bien, ¿̇de qué forma se vincula esta técnica al turismo y la mercadotecnia, si ambos no desarrollan aparatos? Se entiende aquí, que la manipulación, tanto de la naturaleza como del hombre se produce desde la mercadotecnia, a través del turismo y viceversa, de modo de uno depende del otro, ya que, como técnica, se convierten en medio para conseguir un fin, y este fin, no es otro que el económico.

Heidegger (1994) comenta también, que la técnica conduce a la deshumanización, debido a que, de acuerdo con Arias:

En el afán humano de querer conocer, examinar y predecir todo, se ha caído en una idea de sentido antropocéntrica que se caracteriza por la premisa de dominio y control del hombre sobre la naturaleza en aras del bienestar y del progreso; lo que resulta en la inminente pérdida de sentido originario y desencadena la llamada deshumanización. Es justamente la técnica, que, como parte del hombre se vuelve en su contra y lo aleja de su propia esencia para colocarlo en un aparato. (2017, p. 252).

En este sentido, y siguiendo con el argumento de Arias, se tiene que el turismo no está exento de esta deshumanización, en tanto que los servicios turísticos modernos están siendo diseñados para incrementar una suerte de ensimismamiento del ser humano, cuando él mismo se enfrenta a la tecnología que prescinde de sus servicios y en su lugar coloca aparatos, que, por ejemplo, un robot recepcionista le dé la bienvenida al hotel o una aplicación le sirva de guía dentro de un museo.

La mercadotecnia no se queda atrás, ya que el mercado, cada vez más competido ha obligado a analizar minuciosamente el entorno y motiva con el fin de conseguir rendimientos en las organizaciones con y sin fines de lucro. Según L. Alvarado (2013), saber cómo decidimos y, en especial, conocer qué "botón" dentro del cerebro activa la compra, es el sueño de toda empresa. Al respecto, el marketing digital es un ejemplo de algo que ha permeado de forma vertiginosa en la vida cotidiana de las personas; puesto que se ha encargado de aprovechar las tecnologías contemporáneas generando estrategias innovadoras para llegar a los clientes, sobretodo en el actual contexto en el que el confinamiento y el trabajo en casa, producto de una pandemia sin 
precedentes, han empujado a las empresas a desarrollar estrategias empleando todos los medios posibles.

Hoy más que nunca, la deshumanización de la mercadotecnia es toda una realidad, desde el uso los llamados bots de chat, que atienen las veinticuatro horas del día en una página web, hasta la minería de datos que posibilitan el manejar el Big Data como herramienta de predicción, inteligencia artificial, los algoritmos de redes sociales que seleccionan los contenidos que ven sus usuarios trasformando la percepción de la realidad misma, y cada vez un más largo etcétera de técnicas que están desarrollándose año con año. Con el constante avance de las herramientas de comunicación, de procesamiento de información, la reciente adopción del modelo aplicado de las neurociencias, el estado de multidisciplinariedad del marketing ha resaltado la necesidad de plantearse preguntas más reflexivas, en concreto y que atañe a este escrito ¿Qué hace que la mercadotecnia sea mercadotecnia?

La segunda parte de este apartado implica pensar, pero ¿qué es pensar, según Heidegger? No es otra cosa, sino "meditar sobre algo que a cada uno de nosotros atañe directa y constantemente en su propio ser" (1955, p. 1). Es menester detenerse un poco en este punto y distinguir las dos formas o tipos de pensar; el primero de ellos se refiere al pensar calculador, y el segundo al pensar meditativo (reflexión meditativa). El primero cuenta siempre con circunstancias dadas, cuando, por ejemplo, se crea una empresa o se planifica algo; dichas circunstancias, según Heidegger (1955), son consideradas partiendo de una intensión calculada en función de las metas establecidas; esto quiere decir que de antemano se cuentan con determinados resultados. Esto no implica que deba tratarse de cuestiones numéricas, sino que, "el pensamiento que cuenta, calcula; calcula posibilidades continuamente nuevas, con perspectivas cada vez más ricas y a la vez más económicas" (Heidegger, 1955, p. 2). Entonces, este tipo de pensar no se detiene y no alcanza a meditar.

En lo que se refiere al pensar meditativo o reflexión meditativa, aquél al que el hombre suele huir, en tanto que niega la utilidad debido a que carece de tiempo (gracias al pensar calculador). La meditación, dice Heidegger (1955, p. 2) "no sirve para los negocios ordinarios. No 
ayuda en nada a la acción", puesto que reclama mayor esfuerzo. Aquí se debe acotar que cualquier persona puede seguir a su manera y con sus limitaciones los senderos de la meditación para desvelar la esencia de la cosa en cuestión.

En este sentido, tanto el turismo como la mercadotecnia, se han pensado de forma calculadora, debido a que, dada su tendencia económica y medible, implican metas y se esperan resultados en función de su planificación. Eso quiere decir que, para los fines monetarios de los países, resulta poco atractivo el pensar meditativo, ya que la tarea de pensar no deriva en una acción lucrativa.

\section{¿Qué es fenomenología?}

Si bien, la idea de fenomenología no es algo reciente, ya que desde el s. XVII se ha usado este término en pensadores como I. Kant y G. Hegel, y en el s. xx, E. Husserl y sus seguidores retomaron la fenomenología; sin embargo, dice Audi (2004) no pertenece a una escuela o corriente de la filosofía, sino que se trata de un movimiento impulsado en distintas direcciones, teniendo con esto, diferentes significados para diferentes intelectuales.

Al tratarse de un movimiento pensante, es razonable considerar que, a través del tiempo, se han ido conformando doctrinas, inclusive para los discípulos de una de ellas, tal es el caso de Husserl y los fenomenólogos que lo siguieron (Scheler, 2011; Hartmann, 1934; Heidegger, 2012; Sartre, 1989; Merleau-Ponty, 1994), que para ellos, la fenomenología tiene diferentes sentidos.

De una forma -generalizada- se ha argumentado que la fenomenología ensaya describir directamente la existencia de las cosas "tal y como son en sí mismas" (Audi, 2004, pp. 356), dejando de lado su origen de corte psicológico y su explicación de tipo causal. Esto quiere decir que se encarga de responder la pregunta sobre el significado del Ser. De modo que "la fenomenología es en realidad, el estudio de las esencias, pero también intenta situar a las esencias en la existencia" (Audi, 2004, p. 356). 
Con todo, la esencia de la cosa, según Heidegger (2003), no radica en ser un objeto representado, ni se debe delimitar desde la objetividad del objeto ${ }^{1}$, puesto que no existe un camino que lleve a la objetividad de objeto, y de lo independiente a la cosidad 2 o esencia de la cosa. Esto quiere decir que la fabricación o el material del que está hecha la cosa no representa su esencia; por ejemplo, una jarra que es fabricada con vidrio, la cual tiene, evidentemente, forma de jarra y es nombrada como jarra, no significa en ese conjunto la esencia de la jarra, es decir su nombre, forma, función o material no son su esencia. La cosidad de la jarra radica, según Heidegger (2003), no en contener, sino en verter, y mejor dicho en sacrificar, ofrendar y por consiguiente donar.

Ahora bien, esto se comprende cuando se piensa en la cosidad o esencia de las cosas tangibles, pero ¿qué ocurre con la esencia de cosas intangibles? Para intentar esbozar una respuesta, se debe considerar, nuevamente, que la esencia no es equivalente a la condición sine qua non que constituye a la cosa y en este ejercicio, se pensará en turismo y mercadotecnia.

\section{Fenomenología del turismo}

Hablar de la filosofía del turismo no es algo nuevo, puesto que, como binomio de interés investigativo ha tenido ya cierta producción tanto en la literatura de habla inglesa como hispana; y como ejemplo de ello, D. Comic (1989) reconoce la amplitud de temáticas y disciplinas que se han acercado al turismo, sin embargo, resalta también, que el enfoque filosófico del turismo es inexistente. Más tarde, A. Panosso Netto (2008) expresa la necesidad de estudiar al turismo con ayuda de la filosofía. Del mismo modo J. Tribe (2009) enuncia los principios de la filosofía (virtud, verdad y belleza) y con ellos elabora una descripción superficial del turismo; posteriormente, en un acercamiento similar, E. Arias (2012), realizan un profundo ejercicio interpretativo de esos mismos principios aplicados a la epistemología del turismo.

\footnotetext{
${ }^{1}$ Se entiende que la objetividad del objeto en Heidegger, denota un sentido interpretativo en el que se refiere doblemente al objeto mismo, tomado desde su carácter de mero objeto hasta su carácter fenomenológico.

2 Para Heidegger, las palabras con terminación "dad" se refieren a la posibilidad del ser.
} 
Por último, E. Cohen (2018) -a la manera de Tribe (2009)- menciona que los estudios filosóficos del turismo han avanzado, de modo que catalogó la producción en estudios sobre filosofía, ética y teología del turismo; los cuales abarcan tanto a la producción científica como a la propia industria turística.

Ahora bien, en lo que se refiere particularmente a fenomenología y turismo, se tiene que Cohen (1979) hizo un estudio de sentido fenomenológico en el que planteó cinco tipos de experiencias turísticas (1 the recreational mode; 2 the diversionary mode; 3 the experiencial mode; 4 the experimental mode; 5 the existencial mode) basadas en la visión del turista. En este sentido, Panosso Netto (2008) hace un análisis empírico empleando el método fenomenológico para aproximarse a cuestiones esenciales del turismo mediante un estudio de caso; asimismo G. Szarycz (2009) menciona la frecuencia de uso de la fenomenología como método de estudio en investigación turística; por su parte, T. Pernecky y T. Jamal (2010) proponen un argumento de carácter conceptual y metodológico para estudiar la experiencia y comprensión del turismo, desde postulados de E. Husserl y M. Heidegger; más tarde, Arias (2015) propone la visión hermenéutica del turismo, poniendo énfasis en el sentido de la experiencia, todo ello mediante la postura teórica de M. Heidegger; y también, M. Gisolf (2020) se acerca a la fenomenología hermenéutica de M. Heidegger.

En lo que se refiere a la búsqueda de la esencia del turismo, se han hallado argumentos y realizado estudios, los cuales varían en argumentos y fundamentos tanto filosóficos, como teóricos; y para ilustrar esto se tiene que para D. MacCannell (1976) la esencia del turismo es la búsqueda de autenticidad; para Panosso Netto (en Tribe, 2009) la esencia del turismo es la experiencia del turista; la identidad lo es para L. Tejeda (2017); por otro lado, es el amor, la amistad, el respeto y la paz según E. Castillo, Vázquez y Martínez (2016); para el Foro Iberoamericano de Turismo Sostenible (Fits, 2014) es la sostenibilidad, emprendimiento e innovación social; y para Ruiz (2006) e Ighina (2018) es el ocio.

Se puede considerar al ocio, tiempo libre o la sostenibilidad como las condiciones para que exista el turismo, sin embargo, no como su carácter esencial, puesto que la cosa llamada turismo, si 
bien es un fenómeno humano, el disfrute humano es apenas su función. Por otro lado, los servicios turísticos, los cuales facilitan la estancia, al mismo tiempo representan riqueza para quien los presta, se convierten en la técnica del turismo.

La esencia del turismo debe comprender las tres partes de la experiencia: preparación del viaje, la estancia y la vuelta. Ésta última es la que permite la existencia del turismo, y de no ser por ella, sería un fenómeno migratorio. Pues bien, de esas tres partes, el ser humano recoge y guarda lo experienciado y se queda en su interior como conocimiento. Este conocer dará crecimiento y formación al turista, pero no se habla de formación académica o científica, sino humana, de enriquecimiento intelectual.

Ese conocer, como esencia del turismo, se queda depositado en el interior, en las entrañas del hombre. Aquí se debe mencionar que ese conocimiento no es de tipo práctico o aplicable en algún campo, ya que únicamente sirve de referencia para quien vive la experiencia.

Para intentar ilustrar este punto, se tiene la anécdota de que Kant -considerado como el mejor viajero no viajero-, quien no había salido de su localidad, sin embargo, fue un gran conocedor del mundo, debido a que pasaba el tiempo leyendo sobre otras ciudades. Lo relevante de esta historia es el conocimiento o sabiduría que obtuvo el pensador a través de esos viajes-no viajes y que, dicho sea de paso, tienta ahora sobre el concepto de turismo, el cual, según lo anterior, podría no ser imperativo el desplazamiento físico, sino se debe considerar el desplazamiento mental o imaginativo del lector-turista; pero eso no se discutirá en este ensayo.

\section{Fenomenología de la mercadotecnia}

La mercadotecnia ha inspirado reflexiones de pensadores como Deleuze (1999) y Bauman (2007), donde, por lo regular, se ha abordado la cuestión desde una actitud escéptica debido a la clara intencionalidad de las empresas o gobiernos, y la naturaleza de las herramientas empleadas. B. Ardley (2011) utiliza a la fenomenología, con un enfoque crítico, para cuestionar a la teoría mainstream o tradicional de la mercadotecnia, la cual, plantea, ha descuidado el lado humano. Esto debido a que se basa en supuestos cuestionables sobre la naturaleza del hombre y el manejo de la 
práctica. En esta línea metodológica, se tiene que, a modo de una entrevista semiestructurada, se interroga a distintos informantes clave acerca de su experiencia u opiniones sobre un tema para un proyecto público o de iniciativa privada (Lara, et al, 2018); sobre las respuestas a las interrogantes, el investigador procede a realizar las conclusiones del estudio, se busca aprehender la percepción de los entrevistados - para crear un perfil- con el fin de utilizar esa clasificación para generar una estrategia en la que la organización pueda obtener beneficios, capitalizando el conocimiento, en otras palabras, se trata de una investigación de mercados. En palabras de Kotler y Keller (2012, p. 98) la investigación de mercados es "el diseño sistemático, la recolección, el análisis y la presentación de datos y conclusiones relativos a una situación de marketing específica que enfrenta una empresa", lo que se puede comparar con lo que Heidegger llama "el pensar calculador", mencionado en líneas anteriores; por último, esta aplicación de la fenomenología queda a merced de la técnica, como mero cómputo y análisis de datos, es decir, el estudio se restringe a sí mismo, ya que el investigador no es la figura pensante que se encarga de la ejecución de la reflexión, sino que se limita a gestionar el trabajo de campo. En esta aplicación de la fenomenología no accede a la cosa misma.

La esencia de la mercadotecnia ha merecido distintos argumentos, aunque reducidos en número; por ejemplo, para el catalán B. López-Pinto (2001) el elemento central de la mercadotecnia es el intercambio; en tanto que, para el también español, F. García (2015), por medio de "las cuatro pes", reconoce como carácter esencial la seducción; por su parte C. Carranza (2020) defiende a la claridad como el carácter esencial del marketing.

Si bien, el intercambio es el fin de toda empresa que emplea la mercadotecnia, el intercambio real se da entre el cliente o el consumidor final y la empresa misma; aunado a esto, no todos los individuos involucrados en la mercadotecnia de una empresa entran en la dinámica del intercambio.

La claridad, por su parte, es un punto importante en las estrategias de comunicación. En marketing se usa la claridad sólo si ésta se necesita. La intriga, el misterio o la no claridad 
intencionada son herramientas igual de importantes y útiles para los mercadólogos que la misma claridad.

Por último, en lo que se refiere a la seducción también sería considerada como una útil herramienta, como la misma repulsión (Priestman y Smith, 2018).

Ahora es el turno de responder ¿qué es lo que hace que la mercadotecnia sea la mercadotecnia? La palabra mercadotecnia usualmente es empleada para referirse a una sola de sus funciones, ya sea en las ventas, la publicidad, relaciones públicas, etc., lo cual ha enterrado natural y pasivamente el significado real de la mercadotecnia. Para vislumbrar la esencia de la mercadotecnia, se debe tomar en cuenta sus principales componentes, que desde 1960 hasta el día de hoy se han criticado, pero nunca dejado de utilizar o de enseñar en los programas de educación superior. Es, a través de las llamadas 4 P: producto, precio, plaza y promoción (McCarthy y Perreault, 2011), que se crean las estrategias de mercadotecnia, y con ellas, se engloban las partes de una empresa. Desde el precio, hasta los medios por los cuales se distribuye un producto, son decisiones que competen al marketing. Esta unificación que buscan los mercadólogos entre partes tan distintas de una empresa tiene como propósito el control sobre el flujo de información que irradia el proyecto a gestionar. Se trata de controlar la percepción que se genera con esta información, cortando con el fluir natural de las impresiones que tienen los públicos, tanto internos como externos.

El marketing, para los no mercadólogos, se traduce en el reforzar o cambiar creencias. Desde los mismos empleados, clientes, proveedores y colaboradores externos, hasta la misma sociedad, son tomados en cuenta al momento de idear estrategias de mercadotecnia. Son las manifestaciones del marketing, que, como sus esfuerzos por controlar tienen su raíz en la proyección; la cual se toma aquí como el carácter esencial de la mercadotecnia y se puede entender como ampliar una imagen o una realidad, o también como las sombras de la caverna de Platón, un reflejo de los objetos que ilumina el sol, que, aun habiendo un abismo de diferencia entre la sombra y el objeto, son participes de una misma realidad. 


\section{Reflexiones finales}

Más allá de que, tanto el turismo como la mercadotecnia sean considerados como técnicas o herramientas que se usan para incrementar la rentabilidad de un destino o un producto, y que curiosamente $-\mathrm{y}$ depende del caso-, trabajan juntos (turismo y marketing) dado su potencial de incrementar los indicadores económicos.

Es bien sabido que ambos campos (de conocimiento) han permanecido cómodos en las ciencias económico-administrativas, dadas sus características y bondades en la captación de divisas, inclusive se han considerado como estrategias de desarrollo y que a lo largo del tiempo se han institucionalizado; de modo que, para la comunidad científica, plantear perspectivas fenomenológicas es algo que tiene poca relevancia, sin embargo, es importante pensar y re-pensar y así hallar el sentido de dos objetos que han sufrido de reduccionismo intelectual.

Entonces, la esencia del turismo y la mercadotecnia, así como de cualquier cosa, no se limita a su mera finalidad o utilidad, es decir, supera las condiciones sine que non de la cosa; en lo que tiene que ver al turismo, debe evitar ser reducida al ocio o al amor, sino que su carácter fenomenológico (esencia) radica en conocer, debido a que el turista (más allá de la experiencia) conoce el destino y el enriquecimiento o crecimiento intelectual que se genera es de sentido humano y no científico.

Del mismo modo, el carácter fenomenológico de la mercadotecnia tiene su nacimiento en la idea de la proyección. Todo ejercicio de la mercadotecnia tiene como finalidad el controlar y manipular la imagen de una organización a través de distintas instancias más allá de la comunicación. Pero como todo ejercicio de proyección, los objetos proyectados siempre se encuentran más cerca del ser humano, aunque no sean, en rigor, los objetos referidos.

Sin duda, con el pasar del tiempo, el turismo y la mercadotecnia expanden cada vez más el campo en el que se desarrollan, lo que culmina en un asunto que señala la importancia de repensar las bases en las que reposan las suposiciones.

\section{Referencias:}


Alvarado, L. (2013). Brainketing. En: Alvarado, L., Brainketing. Universidad Peruana de Ciencias Aplicadas. Perú.

https://repositorioacademico.upc.edu.pe/bitstream/handle/10757/550871/Brainketing$\underline{\text { Repos.pdf?sequence }=1 \& \text { isAllowed }=\mathrm{y}}$

Ardley, B. (2011). Marketing theory and critical phenomenology: Exploring the human side of management practice. Marketing Intelligence \& Planning. (29). 628-642. 10.1108/02634501111178668.

Arias, E., Castillo M. y Panosso Netto, A. (2012) Análisis de las visiones del Turismo en México. En: Revista $\quad$ Turismo 286-307. https://www.researchgate.net/publication/279668065 Analisis de las Visiones del Turis $\underline{\text { mo en Mexico }}$

Arias, E. (2015) La experiencia existencial como modo de interpretación del turismo. En Revista Hospitalidade, XII(2) 586-599. https://www.revhosp.org/hospitalidade/article/view/598

Altamira, R. y Muñoz, X. (2007) El turismo como motor de crecimiento económico. Anuario Jurídico y Económico Escurialiense, XL 677-710. file:///C:/Users/cernn/AppData/Local/Temp/DialnetElTurismoComoMotorDeCrecimientoEconomico-2267966.pdf

Audi, R. (2004) Diccionario Akal de Filosofía. España: Akal.

Bauman, Z. (2007) Vida de consumo. México: Fondo de Cultura Económica.

Carranza, C. (2020). La esencia de la mercadotecnia. En: Kiwi! D.S. https://kiwiids.com/la-esenciade-la-

mercadotecnia/\#: :text=La\%20mercadotecnia\%20es\%20la\%20creaci\%C3\%B3n,de\%20alg\%C 3\%BAn\%20producto\%200\%20servicio.

Castillo, E., Vázquez, E. y Martínez, F. (2016) Aproximacion al "turismo Consciente", una propuesta ecuatoriana. En: Memoria del X Citurdes Congreso internacional de Turismo Rural y Desarrollo Sostenible, 567-584. https://dialnet.unirioja.es/servlet/articulo?codigo=6122111

Cohen E. (1979) A Phenomenology of Tourist Experience. En: Sociology 13(2) 179-201. DOI: $10.1177 / 003803857901300203$ 
Cohen E. (2018) The philosophical, ethical and theological groundings of tourism - an exploratory inquiry. En: Journal of Ecotourism.

359-382. https://doi.org/10.1080/14724049.2018.1522477

Comic, D. K. (1989), Tourism as a subject of philosophical reflection. The Tourist Review. 44(2) 6-13. https://doi.org/10.1108/eb058016

Deleuze, G. (1999) Conversaciones. España: Ed. Pre-textos.

Fits (2014) La esencia del Turismo: Sostenibilidad, emprendimiento, innovación social... eso y mucho más en Fits Chile. https://ecosostenibleconsultora.wordpress.com/2014/09/24/la-esenciadel-turismo-sostenibilidad-emprendimiento-innovacion-social-eso-y-mucho-mas-en-fitschile/

García, F. (2015). ¿Qué es en esencia el marketing y para qué sirve realmente? En: Las cuatro pes, marketing para todos. http://lascuatropes.com/2015/05/17/que-es-marketing-para-quesirve/

Gisolf, M. (2020) La fenomenología y el turismo. En Turismo y Teoría. http://www.tourismtheories.org/?p=1530\&lang=es

Hartmann, N. (1934) Fundamentos de la ontología. México: F. C. E.

Heidegger, M. (1955) Serenidad. Ed. Tekne. Argentina.

http://gorla.com.ar/DIVAGUES/Serenidad\%20Heidegger/Serenidad.pdf

Heidegger, M. (1994) La pregunta por la técnica. España: Del Serval.

Heidegger, M. (2003) Filosofía, ciencia y técnica. Chile: Universitaria.

Heidegger, M. (2012) Ser y tiempo. España: Trotta.

Ighina, C. (2018) Turismo, tiempo libre y ocio espiritual. Comercio y Justicia. https://comercioyjusticia.info/opinion/turismo-tiempo-libre-y-ocio-espiritual/ Kotler, P. y Keller K. (2012) Dirección de marketing. México: Pearson Educación. Kotler, P.; Bowen, J. T.; Makens, J. y Baloglu, S. (2017). Marketing for Hospitality and Tourism. Boston, MA: Pearson Education. https://digitalscholarship.unlv.edu/hotel fac articles/120/ 
Lara, E., Ocaña, A., Gracia, G., Aguirre, S. (2018) Uso de la fenomenología en la creación de estrategias de comunicación turística para la ciudad de Guayaquil. En: Espirales Revista Multidisciplinaria de Investigación Científica. http://www.revistaespirales.com/index.php/es/article/view/646/html\# ftn1

López-Pinto, B. (2001) La esencia del marketing. Barcelona: Edicions UPC.

Martos, L. (2015) Marketing en el sector turístico. España: Síntesis.

MacCannell, D. (1976) The tourist: A new theory of the leisure class. Schoken Books, published by arrangement with the University of California Press. New York.

McCarthy J. y Perreault, W. (2011). Marketing: planeación estratégica, de la teoría a la práctica. Colombia: McGraw-Hill.

Merleau-Ponty, M. (1994) Fenomenología de la percepción. España: Planeta-Agostini.

Panosso, A. (2008) Filosofía del Turismo: teoría y epistemología. México: Trillas.

Panosso, A. (2009) What is tourism? Definitions, Theoretical Phases and Principles. En: Tribe, J. Philosophical Issues in Tourism. Channel View Publications. UK.

Papadopoulos, S. (1987), "Strategic marketing techniques in international tourism. International Marketing Review. 4(2). 71-84. https://doi.org/10.1108/eb008331

Parra, M. y Beltrán, M. (2016) Estrategias de marketing para destinos turísticos. Ed. Eumed. http://www.eumed.net/libros/libro.php?id=1560

Pernecky, T. y Jamal, T. (2010) (Hermeneutic) Phenomenology in tourism studies. En: Annals of Tourism Research. 37(4) 1055-1075 https://doi.org/10.1016/j.annals.2010.04.002

Priestman S. y Smith A. (2018). Why repulsion is the secret to modern marketing. En: Campaing. https://www.campaignlive.com/article/why-repulsion-secret-modern-marketing/1455777

Ruiz, M. (2006) Reflexiones sobre el ocio como esencia y práctica del turismo. En: OcioGune 2006: el ocio en las disciplinas y áreas de conocimiento: comunicaciones. Aguilar, E. y Rubio, I. (Coords.) España: Universidad de Deusto = Deustuko Unibertsitatea.

San Martín, J. (2002) La estructura del método fenomenológico. Universidad Nacional de Educación a Distancia. Ed. Aula Abierta. España. Disponible en 
https://www2.uned.es/dpto fim/profesores/JSM/RepositorioCV JSM/Libros/5 21 Laestruc turadelmetodofenomenologico.pdf

Sartre, J.-P. (1989). El ser y la nada. México: Alianza Universidad/Losada.

Scheler, M. (2011) La esencia de la filosofía y la condición moral del quehacer filosófico. España: Encuentro.

Szarycz, G. (2009) Some issues in tourism research phenomenology: a commentary. En: Current Issues in Tourism. (12) 47-58. https://doi.org/10.1080/13683500802279949

Tejeda, L. (2017) Identidad debe ser la esencia del turismo. En: Listin Diario. Disponible en https://listindiario.com/economia/2017/09/20/483134/identidad-debe-ser-la-esencia-del$\underline{\text { turismo }}$

Tribe, J. (2009) Philosophical issues in tourism. Channel view publications. UK.

Waldenfels, B. (2017) Fenomenología de la experiencia en Edmund Husserl. En: Areté Revista de Filosofía. 29(2) 409-426. http://dx.doi.org/10.18800/arete.201702.008 